CORRIGENDUM

doi:10.1038/nature 19077

\title{
Corrigendum: Imbalance between pSmad 3 and Notch induces CDK inhibitors in old muscle stem cells
}

Morgan E. Carlson, Michael Hsu \& Irina M. Conboy

Nature 454, 528-532 (2008); doi:10.1038/nature07034

In Fig. 1a of this Letter, the immunofluorescence images for myostatin and follistatin are inaccurate owing to many versions of this figure in multiple revisions of our manuscript. Specifically, the immunofluorescence panels representing anti-myostatin and anti-follistatin staining were duplicated. We would like to thank the anonymous reader who pointed out this error. We have repeated the age-specific immunofluorescence experiments for myostatin and follistatin, and the results confirm our original conclusion that no age-specific differences are detected (Fig. 1). Furthermore, we have performed quantification of the pixel density, and although there are areas with higher and lower signals for these proteins in both young and old muscle sections, the $P$ values suggest no age-specific differences. We thank M. Mehdipour for performing the experiments and analysing the data on age-specific muscle tissue levels of myostatin and follistatin.
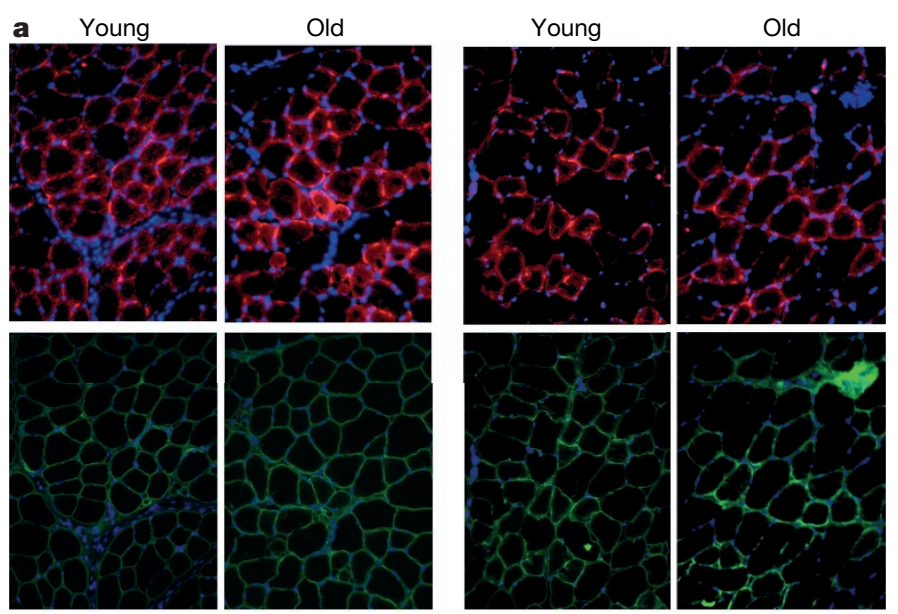

Follistatin Dystrophin Hoechst

Myostatin Dystrophin Hoechst

Figure 1 | This is a repeat of Fig. 1a of the original Letter. Shown are representative images at $\times 20$. Dystrophin immunofluorescence (green) outlines the muscle fibres in $10-\mu \mathrm{m}$ skeletal muscle cryosections that are immunostained for follistatin and myostatin (red); Hoechst (blue) stains all nuclei. $P$ values for mean pixel density of myostatin and follistatin (with rabbit IgG control signals subtracted) show no statistically significant differences between young and old muscle. 